\section{(C) OPEN ACCESS}

\title{
Mucosa-associated invariant T cells link intestinal immunity with antibacterial immune defects in alcoholic liver disease
}

\author{
Antonio Riva, ${ }^{1,2}$ Vishal Patel, ${ }^{1,2,3}$ Ayako Kurioka, ${ }_{1}^{4}$ Hannah C Jeffery, ${ }^{5}$ Gavin Wright, ${ }^{6}$ \\ Sarah Tarff, ${ }^{6}$ Debbie Shawcross, ${ }^{2,3}$ Jennifer M Ryan, ${ }^{3}$ Alexander Evans, ${ }^{7}$ Sarah Azarian, ${ }^{1}$ \\ Jasmohan S Bajaj, ${ }^{8}$ Andrew Fagan, ${ }_{1}^{8}$ Vinood Patel, ${ }^{9}$ Kosha Mehta, ${ }_{1}^{9}$ Carlos Lopez, ${ }^{9}$ \\ Marieta Simonova, ${ }_{10}$ Krum Katzarov $_{1}{ }^{10}$ Tanya Hadzhiolova, ${ }^{10}$ Slava Pavlova, ${ }^{10}$ \\ Julia A Wendon, ${ }^{3}$ Ye Htun Oo, ${ }^{5}$ Paul Klenerman, ${ }^{4}$ Roger Williams, ${ }^{1,2}$ Shilpa Chokshi ${ }^{1,2}$
}

- Additional material is published online only. To view please visit the journal online (http://dx.doi.org/10.1136/ gutjnl-2017-314458)

For numbered affiliations see end of article.

\section{Correspondence to} Dr Shilpa Chokshi, Institute of Hepatology, London Foundation for Liver Research, 111 Coldharbour Lane, London SE5 9NT, UK; s.chokshi@ researchinliver.org.uk

Received 5 May 2017 Revised 14 September 2017 Accepted 15 September 2017 Published Online First 2 November 2017

\section{Linked}

http://dx.doi.org/10.1136/ gutjnl-2017-315284

Check for updates

To cite: Riva A, Patel $\mathrm{V}$ Kurioka $A$, et al. Gut 2018:67:918-930.

\begin{abstract}
Background/aims Intestinal permeability with systemic distribution of bacterial products are central in the immunopathogenesis of alcoholic liver disease (ALD), yet links with intestinal immunity remain elusive. Mucosaassociated invariant T cells (MAIT) are found in liver, blood and intestinal mucosa and are a key component of antibacterial host defences. Their role in ALD is unknown. Methods/design We analysed frequency, phenotype, transcriptional regulation and function of blood MAIT cells in severe alcoholic hepatitis (SAH), alcohol-related cirrhosis (ARC) and healthy controls (HC). We also examined direct impact of ethanol, bacterial products from faecal extracts and antigenic hyperstimulation on MAIT cell functionality. Presence of MAIT cells in colon and liver was assessed by quantitative PCR and immunohistochemistry/gene expression respectively. Results In ARC and SAH, blood MAIT cells were dramatically depleted, hyperactivated and displayed defective antibacterial cytokine/cytotoxic responses. These correlated with suppression of lineage-specific transcription factors and hyperexpression of homing receptors in the liver with intrahepatic preservation of MAIT cells in ALD. These alterations were stronger in $\mathrm{SAH}$, where surrogate markers of bacterial infection and microbial translocation were higher than ARC. Ethanol exposure in vitro, in vivo alcohol withdrawal and treatment with Escherichia coli had no effect on MAIT cell frequencies, whereas exposure to faecal bacteria/ antigens induced functional impairments comparable with blood MAIT cells from ALD and significant MAIT cell depletion, which was not observed in other T cell compartments.
\end{abstract}

Conclusions In ALD, the antibacterial potency of MAIT cells is compromised as a consequence of contact with microbial products and microbiota, suggesting that the 'leaky' gut observed in ALD drives MAIT cell dysfunction and susceptibility to infection in these patients.

\section{INTRODUCTION}

Alcohol-related liver cirrhosis (ARC) induces a state of profound immunodeficiency, known as cirrhosis-associated immunodeficiency syndrome (CAIDS). ${ }^{1-3}$ When patients with alcohol-related liver disease (ALD) present with severe alcoholic hepatitis (SAH), the susceptibility to bacterial infection is further heightened, and infection is observed in nearly $50 \%$ of cases in the short term. ${ }^{4}$ We recently illustrated widespread impairment of antibacterial adaptive $\mathrm{T}$ cells in $\mathrm{SAH}$ and showed restoration of function with immune checkpoint-receptor inhibition. ${ }^{5}$ Interestingly, we did not observe adaptive $\mathrm{T}$ cell dysfunctions in ARC, ${ }^{5}$ suggesting that alternate mechanisms drive immunoparesis in these patients. Infection in ARC precipitates severe complications including septic shock, acute-on-chronic liver failure and multiorgan failure. ${ }^{23}$ Therefore, it is imperative that we delineate common and divergent immune pathways in ARC and SAH to develop specific and targeted immunotherapeutic strategies to reconstitute the host immunity, rather than the continued use of antibiotics in an era of multidrug resistance. ${ }^{6}$

MAIT cells are innate-like CD161-positive antibacterial $\mathrm{T}$ cells considered key for the immune control of gut microbiota, bacterial infection and inflammatory diseases. ${ }^{7-11}$ Initially characterised in the intestinal mucosa, they are now known to be the most predominant population of intrahepatic $\mathrm{T}$ cells and appear in high frequencies in the peripheral blood physiologically. ${ }^{812-14}$ MAIT cells have a semi-invariant $\mathrm{T}$ cell receptor, containing the V $\alpha 7.2-\mathrm{J} \alpha 33$ chain and a diverse $\mathrm{V} \beta$-chain pool. ${ }^{11}$ They recognise riboflavin metabolites of bacterial/fungal origin presented via the major histocompatibility complex (MHC)-related molecule 1 (MR1), ${ }^{15}$ which is inducible upon bacterial infection $^{16}$ or IFN $\gamma$ stimulation ${ }^{17}$ and expressed on various cells, including B cells and monocyte. ${ }^{16}$ Mature MAIT cells are predominantly CD8 single-positive, ${ }^{15} 18$ express the immunoenzyme CD26, the interleukin (IL)-7/IL-18 receptors and respond to both T cell receptor (TCR)-dependent and TCR-independent stimulation. ${ }^{17}{ }^{19}$ Activated MAIT cells perform antibacterial functions by secreting cytokines (IFN $\gamma /$ TNF $\alpha /$ IL-17) and killing MR1-positive infected cells. 811 20-24

Given that MAIT cells defend the host at key locations associated with alcohol-induced liver injury, namely the gut and the liver, we hypothesised that 
What is already known on this subject?

- One of the major and most common complications that patients with alcohol-related liver disease (ALD) face is an increased susceptibility to infection, which can lead to worsening of liver disease and multiorgan failure. Indeed, the inability to contend with bacterial infection is associated with a four-fold increase in mortality, and once the infection is established $30 \%$ of these patients will die within 1 month and a further $30 \%$ by 1 year. To date, we have limited understanding regarding the processes that mediate this immune impairment. Recently, a new subset of innate T cells, known as mucosa-associated invariant T cells (MAIT), have been described as a key component in the arsenal of antibacterial host defences. These are unconventional T cells that respond to bacteriaderived metabolites and are found in large numbers in the liver, intestinal mucosa and peripheral blood. They represent a fundamental sentinel system for the homeostatic control of the gut flora and exert essential roles for the control of bacterial infections. Given that these cells defend the host at key locations associated with alcoholic liver disease, such as the gut and the liver, it is likely that they are intimately involved in the susceptibility to infection observed in patients with ALD. This study is the first report comprehensively describing their role in the defective antibacterial immunity in ALD, which was previously unknown.

What are the new findings?

- A broad spectrum of dramatic quantitative and functional impairments of blood MAIT cells exist in patients with alcohol-related cirrhosis, which is exacerbated in those with severe alcoholic hepatitis possibly signifying an association with disease severity.

- The majority of blood MAIT cells in patients with ALD are deleted and the remaining population exists in a state of 'poised' activation, displaying increased expression of activation markers, but lack of lineage-specific transcription factors and significantly compromised antibacterial cytokine and cytotoxic responses.

- Loss of gut integrity and the increased translocation of gut bacteria, bacterial antigens and metabolic products drive selective MAIT cell depletion and dysfunction resulting in highly compromised antibacterial defence systemically.

- Intrahepatic MAIT cells in ALD are numerically preserved with preferential portal accumulation, despite a significant deficit in blood MAIT cell frequencies, suggesting a key role as biliary firewall protecting the epithelial lining from translocated bacteria.

How might it impact on clinical practice in the foreseeable future?

- The most common cause of death in patients with alcohol-related liver cirrhosis is the development of serious bacterial infections as a consequence of impaired host immune defences. Current guidelines recommend intensive and early antibiotic therapy in these patients. However, this has led to the increasing emergence of multidrug-resistant bacteria. These infections are associated with a higher incidence of septic shock and/or rapid deterioration of liver function and death. As such, there is a pressing need to explore new paradigms for anti-infective therapy, and we believe that host-directed immunomodulatory therapies are a promising approach. The findings from this report highlight MAIT cells as a potential therapeutic target to restore immune competency in ALD. Strategies aimed at restoring the gut barrier, at functional reprogramming of MAIT cells or the use of MAIT cells derived from induced pluripotent stem cells (iPSC-derived MAIT cells) may represent novel attractive immunotherapeutic avenues to allow reconstitution of antibacterial immunity.

they may play an important role in the immunopathogenesis of ALD.

\section{MATERIALS AND METHODS}

\section{Subjects and study design}

The study was performed conforming to the Declaration of Helsinki, with full informed patients' consent and individual ethical approval from all recruiting centres (online supplementary methods).

A. MAIT cell phenotype and function were primarily assessed on the following three cohorts:

1. SAH $(n=13)$ : with Maddrey's discriminant function $\geq 32,{ }^{25}$ excluding patients receiving immunosuppressants prior to/at the time of sampling.

2. ARC $(\mathrm{n}=9)$ : excess alcohol drinkers ( $>80 \mathrm{~g} /$ day $/$ male $>60 \mathrm{~g} /$ day/female) with compensated disease (Child-Pugh $\mathrm{A} / \mathrm{B}$ ) seen as outpatients, excluding patients with $>4$ weeks abstinence, cancer, GI bleeding, untreated sepsis or immunomodulatory treatments.

3. Healthy controls (HCs; $n=13$ ): non-smoking healthy volunteers.

In addition, long-term excess alcohol users undergoing monitored short-term alcohol withdrawal $(\mathrm{n}=35$, paired) were tested at baseline (still drinking) and after 2-5 days of abstinence. Subjects were recruited from the Salvation Army alcohol rehabilitation centre based on Drug and Alcohol Action Team (DAAT) referrals, with exclusion of patients with documented severe mental disorders (DAAT documents, Salvation Army screening and nursing team interviews) and/or unable to give informed consent. Of these 35 patients, five had liver stiffness (measured by FibroScan ' $\mathrm{E}$ ' parameter) above threshold for cirrhosis $(\geq 12)$, while 20 had liver stiffness $<7$.

Bacterial infection was defined as previously shown. ${ }^{5}$

B. To investigate the impact of faecal extracts on MAIT cells: stool samples were collected from 27 patients with ALD (SAH: $\mathrm{n}=7$; ARC: $\mathrm{n}=20$ ) and 12 HCs.

C. To measure MAIT cell frequencies in colon and liver tissue: colon pinch biopsies were obtained from 11 ARC and $10 \mathrm{HCs}$ colon resections; liver biopsies were obtained from explanted livers of five ARC and five healthy liver donors.

Table 1 summarises all patients' characteristics.

\section{Isolation of peripheral blood mononuclear cells (PBMC)}

PBMC were isolated by density gradient centrifugation (Lymphoprep, Alere, Stockport, UK) and cryopreserved in 10\% DMSO (Sigma-Aldrich, Gillingham, UK) as previously described. ${ }^{526}$

\section{Plasma endotoxin and D-lactate}

Surrogate markers of bacterial infection and intestinal transloca$\operatorname{tion}^{27}$ were measured with the Limulus-amebocyte-lysate assay 


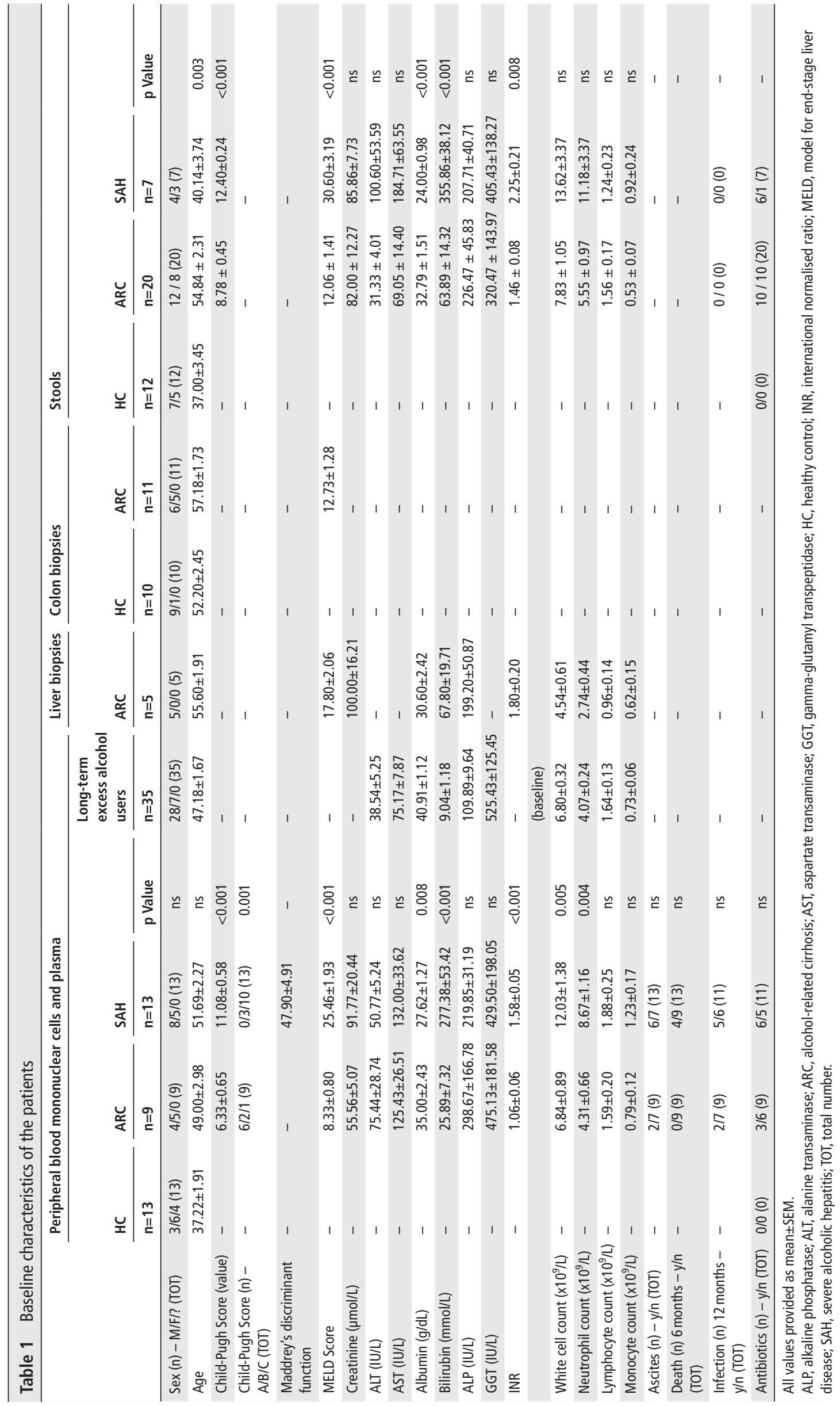


(Pierce/Thermo Fisher Scientific, Paisley, UK) and a colorimetric D-lactate assay (AbCam, Cambridge, UK) respectively.

\section{Preparation of fixed Escherichia coli and faecal extracts of bacterial antigens, toxins and metabolites (FEB)}

Stocks of E. coli DH5 $\alpha$ were grown in RPMI 1640 medium (Gibco/Thermo Fisher Scientific), fixed in BD Cytofix buffer (formaldehyde 4\% in PBS, BD Biosciences, Oxford, UK) (10 min, room temperature (RT)), extensively washed in PBS (Gibco/ Thermo Fisher Scientific) and preserved at $4^{\circ} \mathrm{C}-8^{\circ} \mathrm{C}$ (or $-80^{\circ} \mathrm{C}$ for long-term storage). Stool samples from patients with ALD and HC were homogenised in PBS. Suspensions were clarified with five rounds of centrifugation at increasing speed (1000$1500 \mathrm{rcf}$ ) and duration (1-5 min) to discard debris pellets. Clarified faecal extracts were pelleted, fixed and preserved following the same protocol used for E. coli. All bacterial concentrations were determined by visual counting with a Neubauer-Petroff chamber.

\section{Experimental design and cell cultures}

Cryopreserved PBMCs were defrosted, counted and used for flow cytometry directly or after overnight culture $\left(37^{\circ} \mathrm{C}, 5 \%\right.$ $\mathrm{CO}_{2}$ ) in round-bottom 96-well plates $(300000 / 200 \mu \mathrm{L} /$ well $)$ in RPMI 1640 medium, $10 \%$ pooled heat-inactivated human AB serum (Sigma-Aldrich), $100 \mathrm{IU} / \mathrm{mL}$ penicillin/streptomycin, $2.2 \mathrm{mmol} / \mathrm{L}$ 1-Glutamine, $23 \mathrm{mmol} / \mathrm{L}$ HEPES (all from Gibco/ Thermo Fisher Scientific). Cell recovery and viability were always greater than $90 \%$. Cells were unstimulated or stimulated overnight with fixed E. coli (100 bacteria per cell (BpC)). One hour before adding E. coli, some cultures were (1) preblocked with polyclonal blocking antibody anti-PD1 $(10 \mu \mathrm{g} / \mathrm{mL}$, AF1086, BioTechne/R\&D Systems, Abingdon, UK); (2) preincubated with ARC/SAH plasma (10\%); (3) pretreated with increasing concentrations of ethanol (50-100-250 $\mathrm{mmol} / \mathrm{L})$; or (4) preincubated with ARC/SAH/HC faecal extracts (FEB, $100 \mathrm{BpC}$ ). Brefeldin A (Sigma-Aldrich) was added to cultures used for intracellular flow cytometry 1 hour after E. coli $(10 \mu \mathrm{g} / \mathrm{mL})$.

\section{Immunophenotyping, intracellular staining and functional apoptosis}

MAIT cells were identified by flow cytometry using the following panel: CD3/CD4/CD8/TCR_V $\alpha 7.2 / C D 161$. We measured (1) activation markers and immune checkpoint receptors (CD69/ HLA-DR/PD1/TIM3/LAG3); (2) intracellular cytokines/cytotoxicity markers (IFN $\gamma / \mathrm{TNF} \alpha / \mathrm{IL}-17 /$ GranzymeB/Perforin/CD107a); (3) homing-related markers (beta7-integrin/CCR9/CXCR3/ CX3CR1/CD26); (4) cytokine receptors (IL-7R/IL-18R); (5) proliferation/senescence markers (Ki67/CD57); and (6) transcription factors (ROR $\gamma t / \mathrm{PLZF} /$ Eomes/T-bet). The impact of stool on MAIT cell caspase-dependent apoptosis was assessed by exposing healthy PBMC cultures with FEB (as described above) and measuring apoptosis rates using the Vybrant-FAM PolyCaspase kit (Thermo Fisher Scientific) following the manufacturer's instructions. Online supplementary methods and online supplementary table 1 describe the detailed staining procedure and list all the antibodies used. Samples were acquired and analysed on a FACSCanto-II (BD Biosciences). Levels of expression of all markers of interest were measured both as percentage (\%) of positive cells and as median fluorescence intensity (MFI).

\section{Detection of TCR_Va7.2 (TRAV1-2) by TaqMan PCR}

RNA from PBMC and colon pinch biopsies was extracted in TriReagent (Ambion/Thermo Fisher Scientific) and chloroform
(Sigma-Aldrich) (15 min, RT) followed by isopropanol precipitation (Sigma-Aldrich) (10 min, RT), washed with ethanol (Sigma-Aldrich) $75 \%$ in RNAse-free water (Ambion/Thermo Fisher Scientific), resuspended in RNAse-free water and stored at $-80^{\circ} \mathrm{C}$. RNA was quantified by NanoDrop spectrophotometry (Thermo Fisher Scientific). cDNA was transcribed with QuantiTect Reverse Transcription kits (Qiagen, Manchester, UK). Real-time TaqMan PCR was performed on an ABI 7500 system (Applied Biosystems/Thermo Fisher Scientific) using previously published primers and probe. ${ }^{28}{ }^{29}$ Online supplementary methods describe the detailed TaqMan PCR protocol.

\section{Gene expression profiling}

Public microarray datasets (Gene Expression Omnibus dataset GDS4389, series GSE28619) ${ }^{30}$ were interrogated to measure selected genes of interest in liver tissue from SAH $(n=15)$ and healthy controls $(n=7)$. See online supplementary table 2 for the lists of all queried/analysed genes.

\section{Identification of TCR_Va7.2-expressing cells via immunohistochemistry and imaging}

Immunohistochemistry was performed as previously reported. ${ }^{8}$ In brief, cubes of liver tissue $\left(1-1.5 \mathrm{~cm}^{3}\right)$ were cut, snap frozen in liquid nitrogen and stored at $-80^{\circ} \mathrm{C}$. Tissue was then embedded in Cryoembed (Leica Biosystems, Newcastle upon Tyne, UK). Seven micrometre thick sections were cut using a cryostat (specimen temperature: $-13^{\circ} \mathrm{C}$; chamber: $-20^{\circ} \mathrm{C}$ ) and stained with purified primary antibody anti-TCR_V $\alpha 7.2$ (clone 3C10, BioLegend, London, UK) or IgG1-isotype-control (1 hour, both at $50 \mu \mathrm{g} / \mathrm{mL}$ in PBS) followed by Impress secondary reagent (Vector Laboratories, Peterborough, UK) (30 min, RT). Parenchyma and portal tracts were imaged on a Zeiss Axioskop 40 Microscope with $20-40 \times$ magnifications and measured using AxioVision SE64 V.4.9. Numbers of TCR_V $27.2(+)$ cells $/ \mathrm{mm}^{2}$ were counted. Online supplementary methods describe the detailed staining procedure.

\section{Statistical analyses}

Sample size/power calculations (alpha $=0.05$, beta $=0.20)$ indicated that the numbers of patients used for the main analyses were sufficient to evaluate the main differences. Statistical significance and family-wise alpha for multiple comparisons were set at $p=0.05$. We used (1) Mann-Whitney and Kruskal-Wallis tests with Dunn's multiple comparisons correction for independent samples; (2) paired Wilcoxon test for paired samples; (3) $\chi^{2}$ test for categorical variables; and (4) Spearman's rho ( $\rho$ ) or Pearson's $\mathrm{r}$ coefficients for correlations as appropriate. E. coli stimulation or pretreatment with patient plasma/ethanol/faecal extracts were evaluated by absolute delta change or fold change versus unstimulated or untreated samples as appropriate. Statistics were calculated with Microsoft Excel, SPSS V.21 and GraphPad Prism.

\section{RESULTS}

\section{Patient characteristics}

All baseline clinical characteristics are summarised in table 1 . As expected, SAH patients were significantly sicker than ARC and had greater propensity to bacterial infection within 1 year of recruitment (OR $2.92,95 \%$ CI 0.41 to 20.90 ): approximately half of SAH developed bacterial infection (5/11) compared with only a fifth of ARC (2/9). One-third of patients with SAH died within 6 months of recruitment, whereas no ARC patients died during the study period. 
A

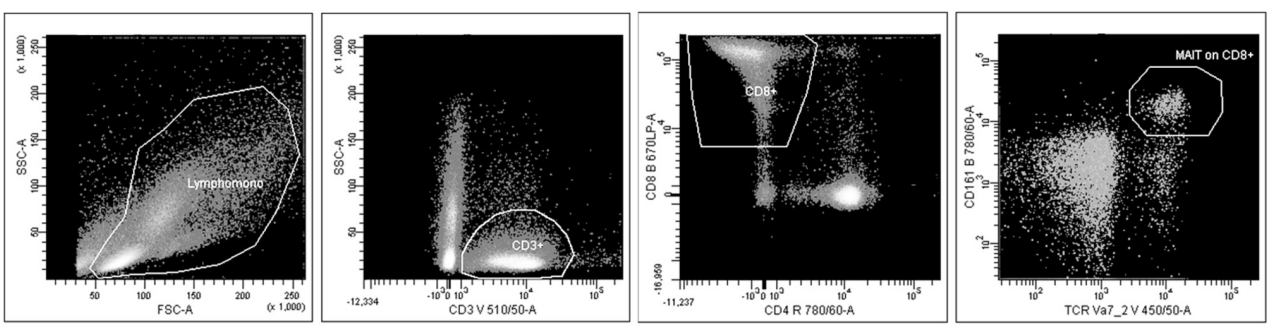

B
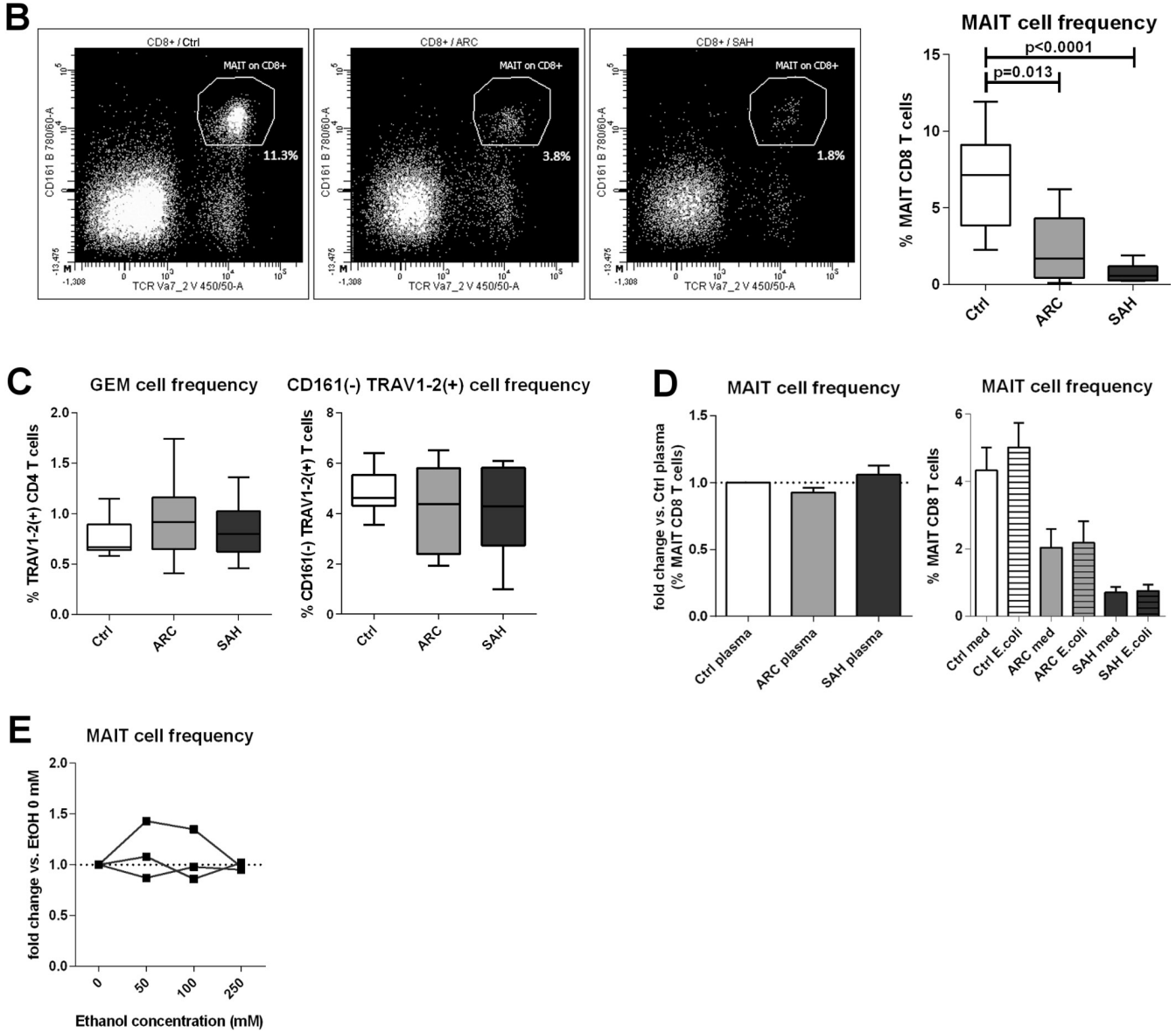

Figure 1 Blood MAIT cell depletion in patients with ALD. (A) Gating strategy to identify MAIT cells by FACS. (B) Blood MAIT cell frequencies in controls $(n=13)$, ARC $(n=9)$ and SAH ( $n=13)$ patients. (C) MAIT cell depletion is selective, as TCR_V $\alpha 7.2(+)$ GEM cells and TCR_V $\alpha 7.2(+) / C D 161(-)$ are unchanged (counts as above). (D) No MAIT cell depletion in healthy PBMC treated with control/ALD plasma (ARC: $n=5 ; S A H: n=5)$ or blood MAIT cells from controls $(n=9), A R C(n=9)$ and SAH $(n=9)$ stimulated with high bacterial load. (E) Lack of MAIT cell depletion upon in vitro ethanol treatment of healthy PBMC. ALD, alcohol-related liver disease; ARC, alcohol-related cirrhosis; FACS, flow cytometry; GEM, germline-encoded mycolyl lipid-reactive T cells; MAIT, mucosa-associated invariant T cells; PBMC, peripheral blood mononuclear cells; SAH, severe alcoholic hepatitis.

\section{Marked selective depletion of blood MAIT cells with intrahepatic MAIT cell preservation in ALD}

MAIT cells were identified as TCR_V $27.2(+) / C D 161($ hi) within the CD8 T cell population ${ }^{11}$ (figure $1 \mathrm{~A}$ ). Blood MAIT cells were significantly reduced in ARC patients compared with controls $(p=0.013)$ and even more dramatically in SAH $(p<0.0001)$ (figure 1B). Interestingly, other TCR_V $\alpha 7.2(+) \mathrm{T}$ cells ${ }^{28}$ were not depleted, and there was no proportional increase of CD161(-) CD8 T cells, suggesting this effect was specific to MAIT cells and not due to CD161 downregulation (figure 1C).

To investigate the mechanisms inducing MAIT cell depletion in ALD, we cultured control/ALD PBMC with fixed E. coli (100 $\mathrm{BpC}$ ) and also control PBMC with ALD/control plasma; ALD plasma contained significantly higher levels of bacterial products than controls (online supplementary figure 1). We found that none of these challenges induced MAIT cell deletion (figure 1D). We did, however, identify a trend for an association between increasing plasma levels of D-lactate (a surrogate marker of gut permeability and intestinal translocation) and reduced blood MAIT cell frequencies in patients with ALD overall (online supplementary figure 1). Additionally, exposing control PBMC to ethanol in vitro $(50-100-250 \mathrm{mmol} / \mathrm{L})$ had no direct MAIT cell toxicity (figure $1 \mathrm{E}$ ), and ethanol withdrawal in vivo did not induce quantitative MAIT cell recovery in excess alcohol users undergoing short-term abstinence (online supplementary figure 2).

We hypothesised that the observed depletion of blood MAIT cells may be due to liver relocation and to assess this 
A

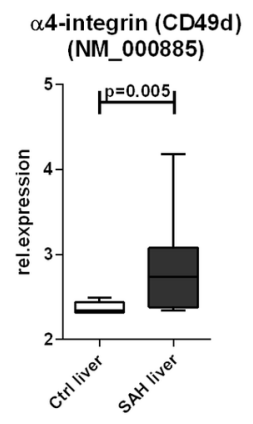

$\alpha 4$-integrin (CD49d) (BG532690)

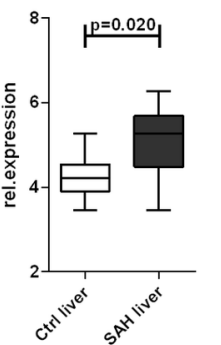

$\alpha$ E-integrin (CD103) (NM_002208)

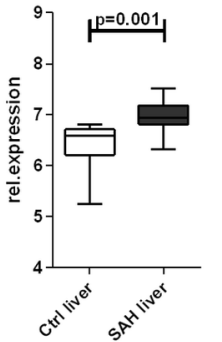

B

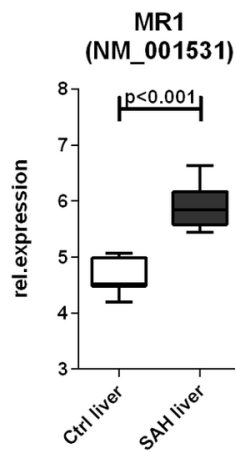

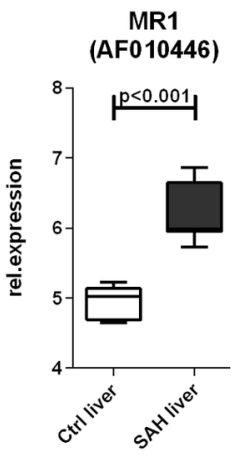

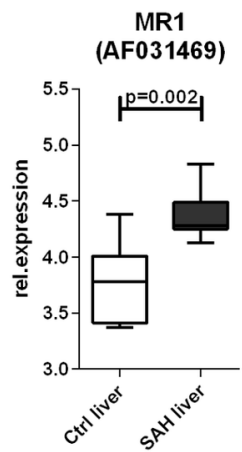

CXCL10 (IP10)

(NM_001565)
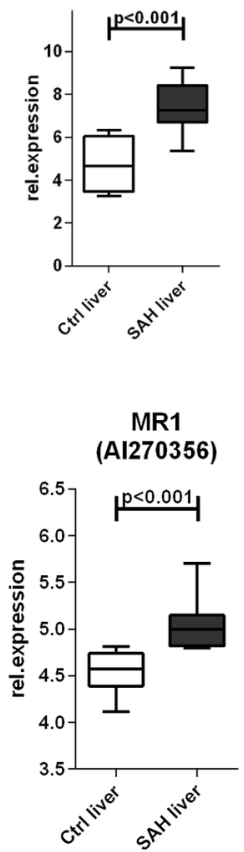

C

Normal
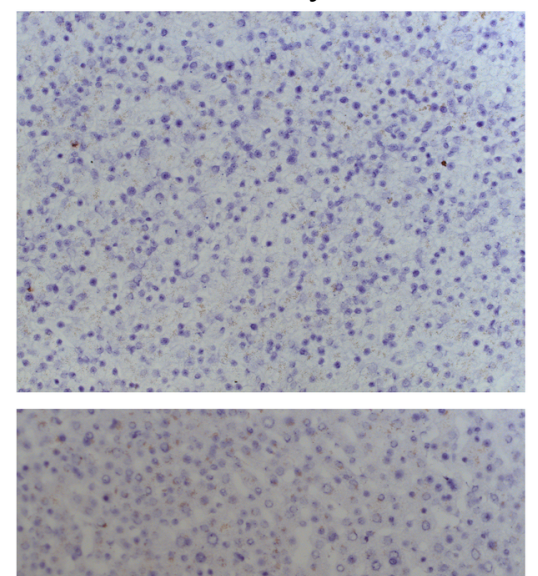

ALD

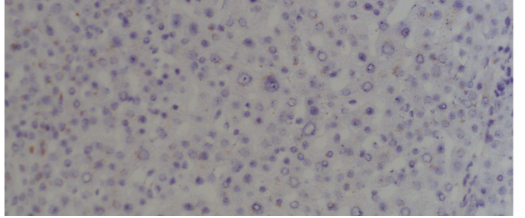

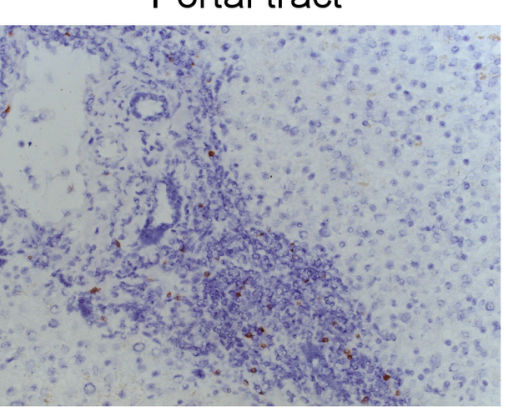

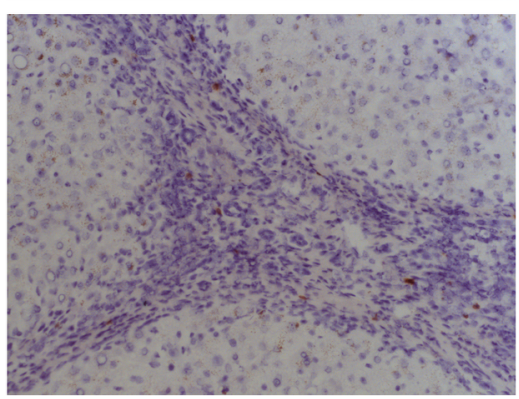

Liver-tissue MAIT cells
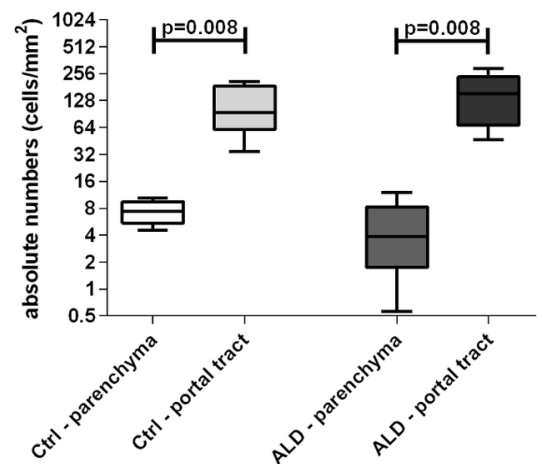

Figure 2 Preservation of MAIT cells in the liver in ALD. Intrahepatic hyperexpression of homing markers (A) and MR1 (B) within total liver tissue, public microarray dataset analysis (Ctrl: $n=7 ; S A H: n=15)$. (C) Liver parenchymal and portal TCR_V $\alpha 7.2$ (clone 3C10) immunohistochemistry to identify intrahepatic TCR_V $\alpha 7.2(+)$ cells; fresh-frozen biopsies from ALD livers $(n=5)$ and healthy liver donors $(n=5)$. ALD, alcohol-related liver disease; MAIT, mucosa-associated invariant T cells; MR1, MHC-related molecule 1; SAH, severe alcoholic hepatitis.

we evaluated the expression of liver homing markers on blood MAIT cells and intrahepatically. We found comparable expression of these markers (total beta7-integrin, CXCR3, CCR9 and CX3CR1) on blood MAIT cells between ALD and controls, and blood MAIT cells from all groups always expressed high levels of IL-7R, IL-18R and CD26/DPP4 as expected ${ }^{13} 31$ (online supplementary figure 3). Conversely, microarray data revealed that intrahepatic alpha4/alphaE integrins (individual beta7-integrins, $\mathrm{p}<0.02$ ) and also T cell chemoattractant CXCL10 (CXCR3-ligand, $\mathrm{p}<0.001$ ) were hyperexpressed in SAH liver tissue compared with controls (figure 2A). Notably, MR1 expression, inducible upon bacterial exposure and relevant to MAIT cell engagement and killing of infected MR1-expressing cells, was also considerably stronger in SAH liver tissue $(\mathrm{p}<0.002)$ (figure $2 \mathrm{~B}$ ). Taken together these data reveal that there are homing signals in the liver that may facilitate intrahepatic MAIT cell relocation or retention in ALD.

We then enumerated TCR_V $\alpha 7.2(+)$ cells in fresh-frozen liver sections from ARC and healthy liver donors and in colon pinch biopsies from ARC patients and controls. Absolute 
numbers of intrahepatic TCR V $\alpha 7.2(+)$ cells in liver parenchymal or portal regions were comparable between patients and controls, with similar greater portal accumulation in both groups $(\mathrm{p}=0.008)$ (figure 2c). TaqMan PCR failed to detect TCR Vo7.2/TRAV1-2 transcripts in colon pinch biopsies (but not in PBMC, used as a positive control) (data not shown), suggesting that MAIT cells may distribute preferentially in locations other than the colonic mucosa.

These data show that MAIT cells are not deleted in the liver of patients with ALD, although being dramatically depleted in their peripheral blood.

\section{Blood MAIT cells are phenotypically hyperactivated but functionally deficient in ALD}

Compounding their significant depletion in the systemic circulation, the remaining population of blood MAIT cells in patients with ALD had a marked hyperexpression of canonical activation markers (CD69: $p<0.008$; HLA-DR: $p<0.03$ ) (figure 3A). A comparable hyperactivated status was induced by treating control PBMC with ALD plasma $(\mathrm{p}<0.008)$ (figure 3B), but not with increasing concentrations of ethanol in vitro (figure $3 \mathrm{C}$ ). CD69 can suppress $\mathrm{T}$ cell functions through binding with its putative ligand Galectin 1 (Gal1), ${ }^{32-34}$ and we found that Gal1 expression was stronger in $\mathrm{SAH}$ liver tissue than controls $(\mathrm{p}<0.001)$ (figure 3D), suggesting greater ability of CD69-mediated intrahepatic MAIT cell inhibition in these patients.

We then assessed whether MAIT cells exhibited signs of exhaustion and immune senescence and found no evidence of this phenotype in ALD: expression of immune checkpoint receptors (PD1/TIM3/LAG3) on blood MAIT cells was comparable in patients and controls (figure 3E); culture of MAIT cells with anti-PD1 antibodies did not modulate MAIT cell phenotype or function (data not shown); furthermore, proliferation or senescence markers (Ki67/CD57) were comparable on blood MAIT cells in all subjects (figure $3 \mathrm{~F}$ ).

We next measured MAIT cell antibacterial cytokine production (IFN $\gamma / \mathrm{TNF} \alpha / \mathrm{IL}-17$ ) and cytotoxic capabilities (GranzymeB/ CD107a/Perforin) in response to E. coli challenge. Starting from very low cytokine production in basal/unstimulated conditions (figure 4A-C), E. coli stimulation induced similar levels of MAIT-specific IFN $\gamma$ and TNF $\alpha$ in all groups $(p<0.008)$ (figure 4A,B), but IL-17-producing MAIT cells were only found in controls: ARC patients had a weaker IL-17 response and this was completely absent in SAH $(p=0.013)$ (figure 4C).

With regards to cytotoxic potential, MAIT cells from all three groups expressed quantifiable basal levels of GranzymeB, CD107a and Perforin, which were marginally greater in ALD patients (both ARC and SAH) than controls (figure 4D-F). Upon E. coli challenge, GranzymeB(+) MAIT cells increased significantly only in controls $(p=0.004)$. GranzymeB induction was weaker in ARC $(\mathrm{p}=0.065)$ and virtually absent in SAH $(\mathrm{p}=0.002)$ compared with controls (figure 4D). The magnitude of GranzymeB responses correlated directly with blood MAIT cell frequencies $(\rho=0.673, p<0.001)$ and inversely with MAIT cell expression of activation markers overall (MAIT/CD69_MFI: $\rho=-0.426, p=0.048 ;$ MAIT/HLA-DR $\%: \rho=-0.558, p=\overline{0} .007$; MAIT/HLA-DR_MFI: $\rho=-0.523, p=0.012)$. E. coli-responsive CD107a(+) MĀIT cells increased significantly in all subjects $(p<0.016)$, but the magnitude of this induction was reduced in both ARC $(\mathrm{p}=0.061)$ and SAH $(\mathrm{p}=0.034)$ compared with controls (figure 4E). Perforin(+) MAIT cells changed comparably in all subjects in response to E. coli (figure 4F). In few patients with SAH, we observed a possible trend for a correlation between E. coli-stimulated cytotoxic potential of MAIT cells and increased plasma markers of bacterial translocation (CD107a induction vs D-lactate in SAH: $r=0.985, p=0.015$ ).

\section{MAIT cells have defective expression of innate-like/type-17 transcription factors in ALD}

Innate-like features and functions by MAIT cells require the expression of transcription factors RORC/ROR $\gamma$ t, ZBTB16/ PLZF, Eomes and T-bet.

We found that expression of RORC/ROR $\gamma$ t and ZBTB16/PLZF was weaker (MFI) and rarer (\% frequency) in blood MAIT cells from patients with ALD, particularly in SAH $(p<0.006)$ (figures 5A and online supplementary figure 4). MAIT cells from SAH also expressed less Eomes and T-bet, although not significantly (online supplementary figures $4-5$ ). These results paralleled the dramatic depletion of blood MAIT cells and the functional defects (IL-17/GranzymeB/CD107a) observed in the remaining blood MAIT cells in patients with ARC/SAH. Notably, microarray data from SAH liver tissue also showed similar features as those observed in blood MAIT cells, with reduction of RORC/ROR $\gamma t(p<0.001), Z B T B 16 /$ PLZF $(p=0.039)$ and also Eomes $(\mathrm{p}<0.001)$ compared with healthy liver tissue (figure 5B).

\section{FEB alter MAIT cell frequencies, phenotype and functions}

Plasma endotoxin and D-lactate were elevated in our patients with ALD, confirming increased intestinal permeability and endotoxemia in these groups (online supplementary figure 1). To test the impact of increased bacterial translocation on MAIT cells, we treated control PBMC with FEB from ALD patients and controls and measured MAIT cell frequency, phenotype and function in basal and E. coli-stimulated samples.

Exposure to FEB from ALD stool extracts induced MAIT cell depletion compared with untreated samples $(p<0.001)$ and also compared with HC stools $(\mathrm{p}<0.025)$ (figure $6 \mathrm{~A})$. This depletion was specific for MAIT cells, as total CD8(+) and total CD3(+) cells were not reduced upon exposure (online supplementary figure 6). Functional apoptosis measured by caspase activation in MAIT cells was always minimal and comparably low in in all stool groups and medium alone (figure 6A).

Expression of MAIT cell activation markers CD69 and HLA-DR was approximately 1.5 -fold stronger and more frequent in samples treated with FEB compared with medium alone $(\mathrm{p}<0.016)$ (figure $6 \mathrm{~B})$. Compared with controls, induction of CD69 was weaker with FEB from patients with ALD but HLA-DR induction was stronger (figure 6B). Exposure to FEB also suppressed MAIT cell antibacterial cytokine responses: E. coli-responsive MAIT cells producing IFN $\gamma$, TNF $\alpha$ or IL-17 were less frequent with ARC or SAH extracts (but not control) $(\mathrm{p}<0.012)$ (figure 6C).

FEB also had a significant impact on the cytotoxic capacity of MAIT cells, with $50 \%$ reduction of GranzymeB $(+)$ MAIT cell frequency $(p<0.008)$ and $30 \%-60 \%$ drop in intracellular granule content $(\mathrm{MFI})(\mathrm{p}<0.008)$. This effect was stronger with SAH extracts (figure 6D). Perforin content was also reduced by approximately $10 \%$ in healthy MAIT cells upon FEB exposure $(\mathrm{p}<0.01)$ (figure 6D), while CD107a expression and responses to $E$. coli were unaffected by FEB (figure 6D).

\section{DISCUSSION}

Our work describes for the first time the characterisation of MAIT cell dysfunctions in the immunopathogenesis of ALD. We reveal a broad spectrum of dramatic quantitative and functional 
A CD69(+) MAIT cells
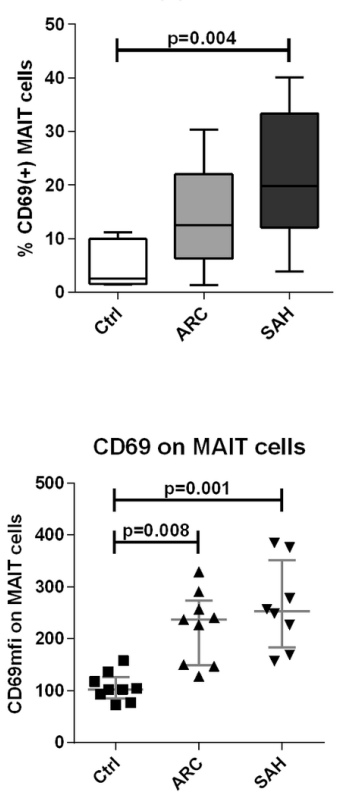

HLA-DR(+) MAIT cells

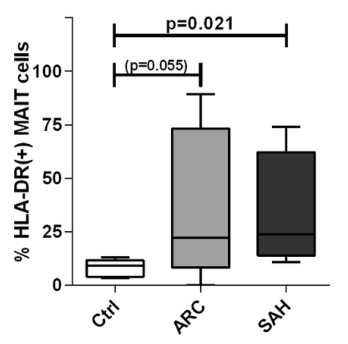

HLA-DR on MAIT cells

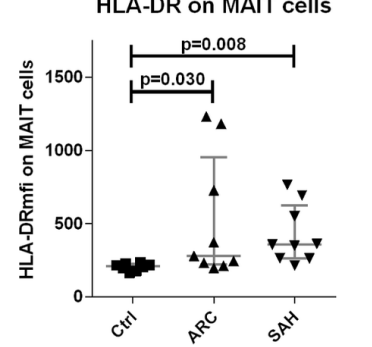

B CD69 expression with ALD plasma
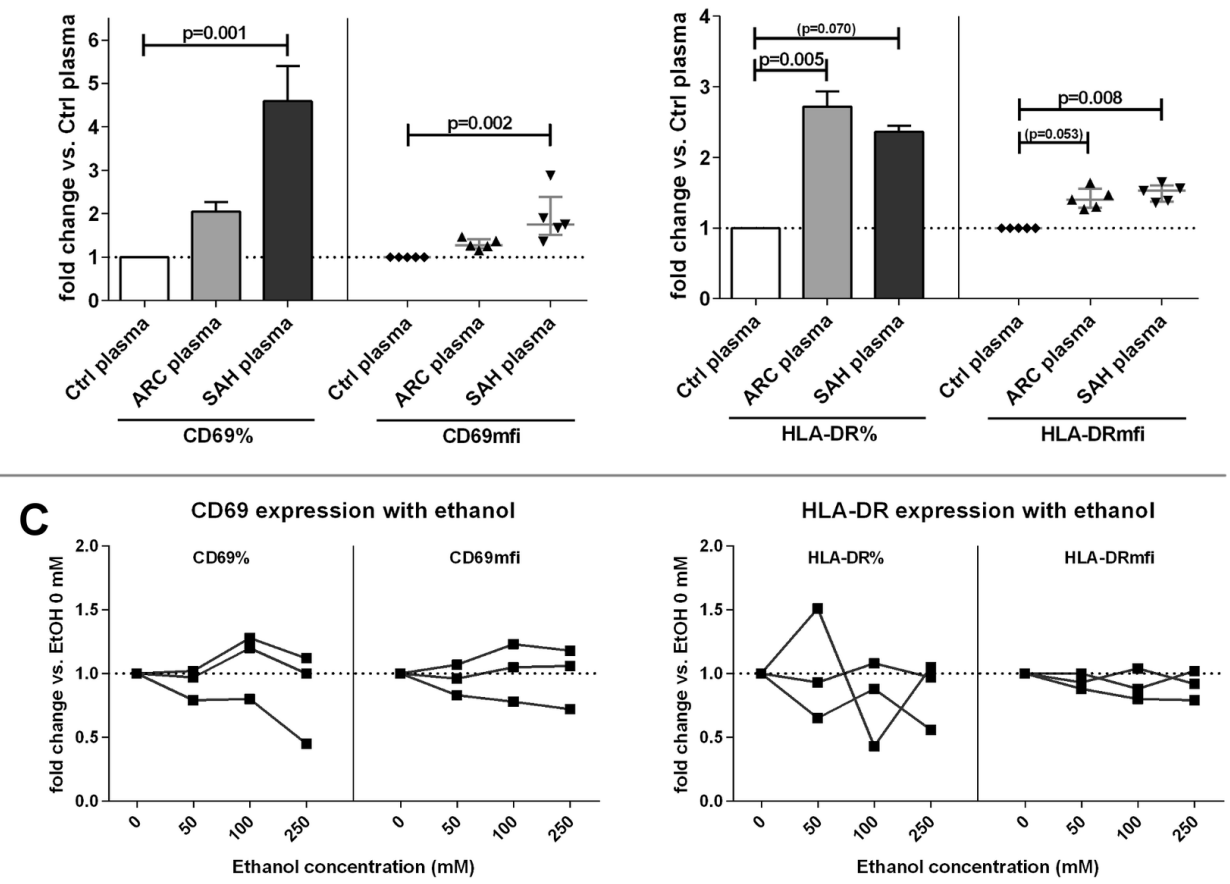

D Galectin 1 (LGALS1) (NM_002305)

E
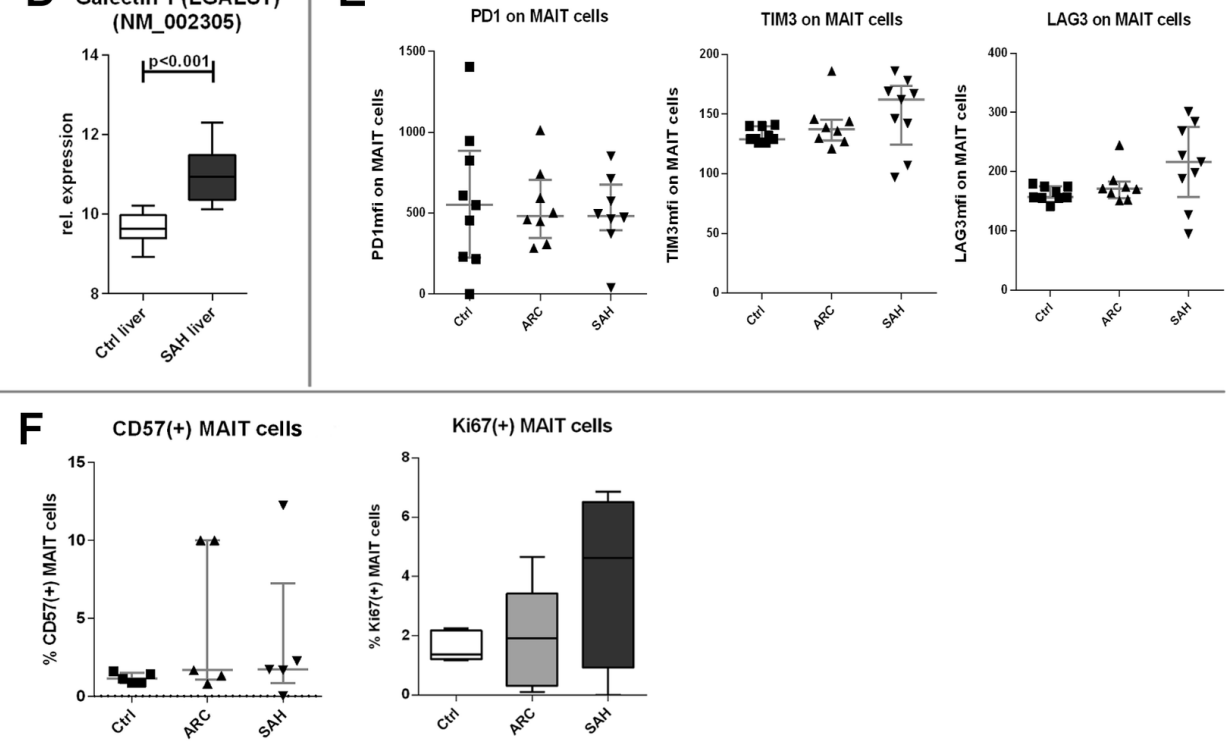

Figure 3 Blood MAIT cells in ALD display a hyperactivated phenotype. (A) CD69/HLA-DR hyperexpression by ALD blood MAIT cells compared with controls ( $n=9$ for all groups). (B) ALD plasma (ARC plasma: $n=5$; SAH plasma: $n=5$ ) can increase CD69/HLA-DR expression in healthy PBMC compared with control plasma. (C) MAIT cell activation status is not induced by in vitro ethanol treatment of healthy PBMC. (D) Gal1 expression is increased in SAH liver tissue (control: $n=7$; $S A H: n=15$ ), public microarray dataset analysis. (E) Immunoinhibitory checkpoints are not upregulated on hyperactivated blood MAIT cells in ALD ( $n=9$ for all groups). (F) Comparable levels of cell proliferation/senescence markers (Ki67/CD57) between groups ( $n=5$ for all groups). ALD, alcohol-related liver disease; ARC, alcohol-related cirrhosis; Gal1, Galectin 1; MAIT, mucosa-associated invariant T cells; PBMC, peripheral blood mononuclear cells; SAH, severe alcoholic hepatitis.

impairments, both in patients with ARC and particularly in those with SAH, possibly signifying an association with disease severity.

We also show for the first time that intestinal bacterial antigens and metabolites drive selective MAIT cell depletion (not affecting CD8/CD3 T cells) and impair MAIT cell antibacterial functionality. This suggests that in patients with ALD, where loss of gut integrity is well characterised, ${ }^{35}$ the increased translocation of gut bacteria, bacterial antigens and metabolites could be detrimental to gut MAIT cells locally and result in systemic depletion of blood MAIT cells and decreased antimicrobial efficacy in the remaining circulating population. In fact, MAIT cell alterations have also been described in other conditions including inflammatory bowel disease (IBD), obesity and diabetes ${ }^{79} 10$ known to harbour gut barrier defects and increased intestinal permeability. While faecal extracts from both ALD patients and controls had effects on MAIT cells overall, there was a greater functional impact from patients with ALD. This may be due to the 
A

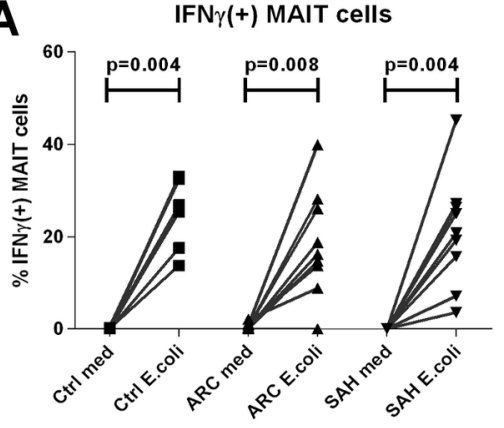

B

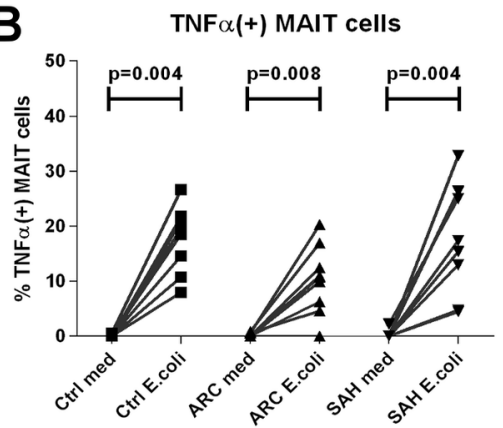

C

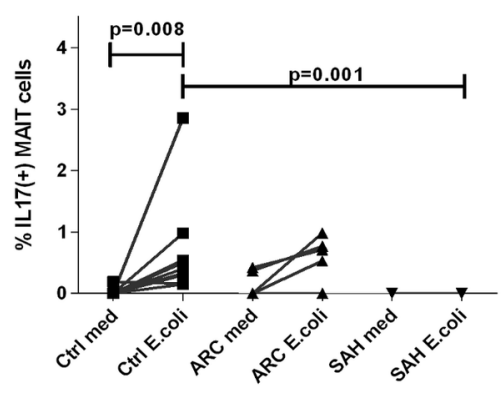

IFN $\gamma(+)$ MAIT cells
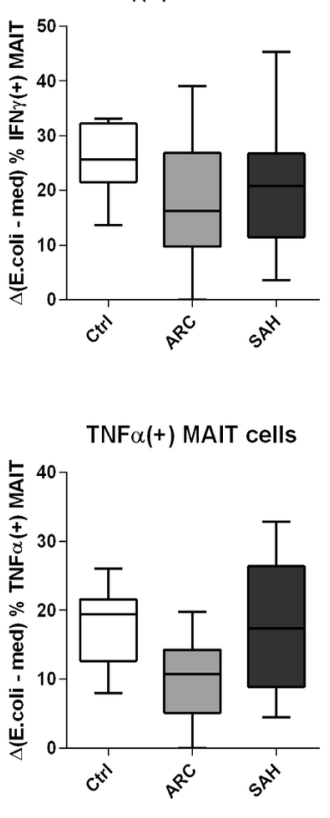

IL17(+) MAIT cells

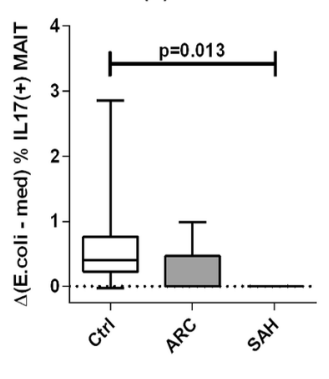

D

Granzyme B(+) MAIT cells

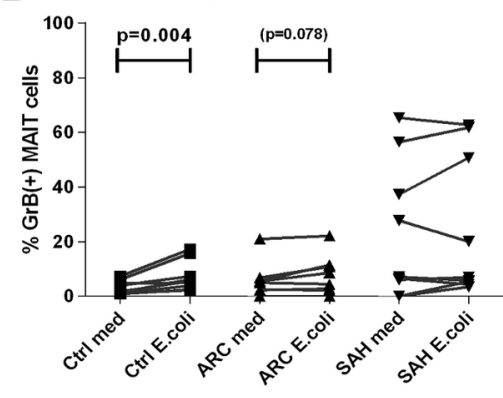

Granzyme B(+) MAIT cells E.coli induction

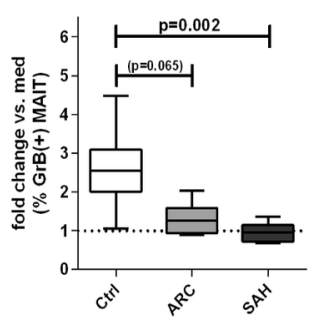

E
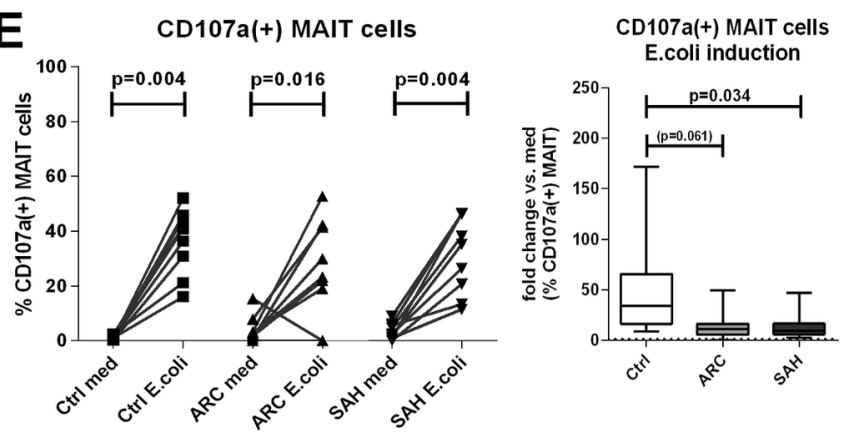

$\mathbf{F}$

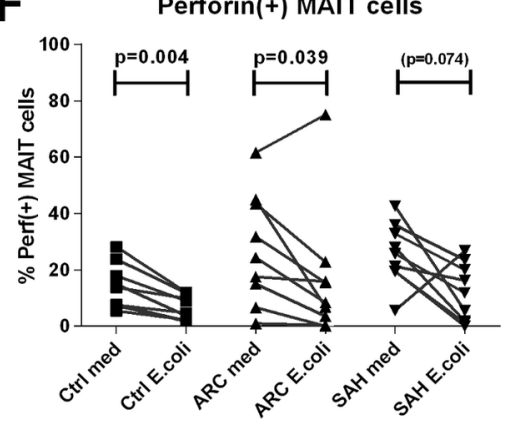

Figure 4 Blood MAIT cells show defective antibacterial cytokine/cytotoxic responses in ALD. (A)-(C) MAIT-specific IFN $\gamma$, TNF $\alpha$ and IL-17 responses upon in vitro Escherichia coli stimulation of ALD/control PBMC, absolute frequencies, and $\triangle$-changes versus unstimulated samples. (D)-(F) MAIT cell GranzymeB, CD107a and Perforin responses upon in vitro E. coli stimulation of ALD/control PBMC; absolute frequencies and fold-changes versus unstimulated samples ( $n=9$ for all groups). ALD, alcohol-related liver disease; ARC, alcohol-related cirrhosis; IFN, interferon; IL, interleukin; MAIT, mucosa-associated invariant T cells; PBMC, peripheral blood mononuclear cells; SAH, severe alcoholic hepatitis; TNF, tumour necrosis factor.

known bacterial dysbiosis observed in patients with ALD. ${ }^{3} 3738$ Physiologically, the intestinal wall is intact, acting as a defensive barrier against the penetration of bacterial products, ${ }^{35} 36$ and mesenteric clearance of translocated bacteria and products is effective. ${ }^{39}$ Conversely, the breakdown of the intestinal barrier in ALD further drives the translocation of macromolecules, microbial products and microbiota, ${ }^{40}$ and the loss of mesenteric bacterial clearance facilitates direct contact with immunocytes. ${ }^{39}$ Moreover, in ALD, it is well described that dysbiosis and microbiota composition are further detrimental to gut barrier integrity due to the increased production of toxic bacterial metabolites such as phenolic compounds, which increase inflammation and directly drive intestinal permeability. ${ }^{38}$

Several other mechanisms could also have been responsible for the observed MAIT cell depletion during ALD, including direct ethanol toxicity or loss of detection due to activation-related CD161 downregulation. ${ }^{41}$ We excluded these hypotheses experimentally and our data suggest that MAIT cell depletion is a direct consequence of contact with gut bacteria, bacterial antigens and metabolites due to loss of gut integrity and increased gut permeability as likelier pathogenic mechanisms, possibly through activation-induced cell death. ${ }^{42}$ Our investigation excludes caspase-dependent apoptosis as the driving cause of MAIT cell loss in our study and we believe that other pathways of both programmed and accidental caspase-independent cell death may be at play such as autophagy, necroptosis, pyroptosis or necrosis. ${ }^{43}$ The processes by which MAIT cells die have not been fully elucidated in the literature and this does require more in-depth investigations.

It has been suggested that recent excess drinking may cause a significant decrease in circulating lymphocyte levels in alcohol users compared with those without recent drinking. ${ }^{44}$ As all the patients in the current study were actively drinking, this could be a contributing factor to the observed MAIT cell reduction. To investigate this, we evaluated MAIT cell frequencies in a cohort of long-term heavy actively drinking alcohol abusers undergoing monitored short-term alcohol withdrawal at baseline, when they were actively drinking, and after 2-5 days of monitored alcohol abstinence and we observed no changes in MAIT cell levels. While these results give further support to our conclusion that ethanol does not impact MAIT cell frequencies, they do not directly address the possibility that recent and excessive drinking 
A RORCIRORYt(+) MAIT cells

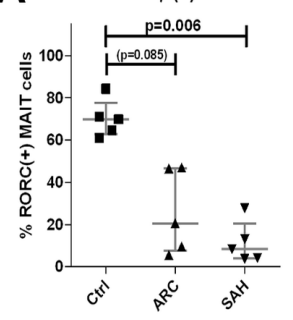

ZBTB16/PLZF(+) MAIT cells
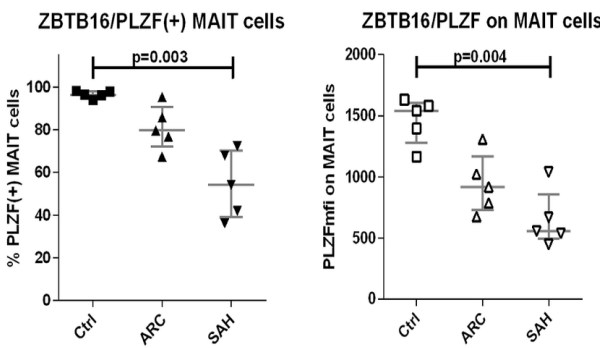

B
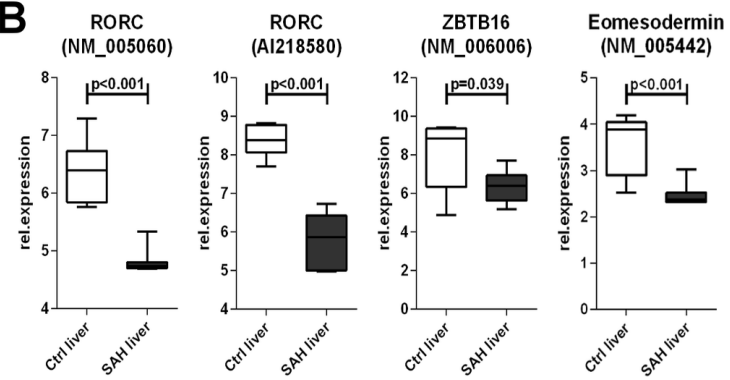

Figure 5 Defective expression of innate-like/type-17 transcription factors by blood MAIT cells and intrahepatically in ALD. (A) Blood MAIT cells from ALD display reduced expression of RORC and PLZF compared with controls ( $n=5$ for all groups). (B) SAH liver tissue $(n=15)$ underexpresses RORC, PLZF and Eomesodermin compared with healthy liver tissue $(n=7)$. ALD, alcohol-related liver disease; ARC, alcoholrelated cirrhosis; MAIT, mucosa-associated invariant T cells; SAH, severe alcoholic hepatitis.

(especially in patients with SAH) causes MAIT cell depletion. We believe that further studies are needed to clarify whether long-term abstinence can improve MAIT cell alterations in ALD, possibly via restoration of gut integrity. Leclercq $e t$ al $l^{38}$ showed that 3 weeks of alcohol abstinence can reduce (but not eliminate) gut permeability in patients with ALD, and this is relevant when considering that even long-term abstinence only partially restores immunity in $\mathrm{ALD}^{3845}$ and vulnerability to infection still remains in some of these patients. ${ }^{45}$

We found that intrahepatic MAIT cells in ALD were not deleted, in spite of blood MAIT cell depletion. Moreover, beta7-integrins and chemoattractant CXCL10 were increased in SAH liver tissue compared with controls, suggesting stronger $\mathrm{T}$ cell recruitment potential in these patients. Intrahepatic MAIT cells showed preferential portal accumulation, and we also observed that SAH liver tissue strongly expressed MR1 compared with controls, suggesting greater potential of MAIT cell TCR engagement in these patients. MAIT cell preservation in peripheral tissues despite blood depletion has been previously described, ${ }^{8234246}$ and Jeffery et $a l^{8}$ showed that intrahepatic MAIT cells express more gut homing beta7-integrins and CXCR3 than blood MAIT cells and more CXCR3 in patients compared with controls. Liver and gut acquire similar homing receptor profiles in pathological conditions, ${ }^{47}$ possibly allowing redistribution of immunocytes including MAIT cells during pathological inflammation following gradients of physiological chemoattractants such as CXCL10, which are upregulated in many types of inflammation and in chronic liver diseases regardless of aetiology. ${ }^{48}{ }^{49}$ We believe that several cell subsets are recruited to the inflamed liver in patients with ALD, that MAIT cells may participate to this same enterohepatic recirculation and that consequently they can be at least numerically maintained in ALD livers although being deleted in the peripheral blood. MAIT cell portal accumulation has been suggested as indicative of their key role as biliary firewall protecting the epithelial lining from translocated bacteria, ${ }^{8}$ and the observed MR1 upregulation would seem to further support this, as it is known to occur upon bacterial uptake, ${ }^{16}$ which is likely increased in patients with increased gut permeability. Intrahepatic MAIT cell preservation and increased MR1 expression in ALD livers may also favour intensified MAIT-mediated tissue damage, as MAIT cells can kill MR1-expressing cells. ${ }^{811}{ }^{20-24}$ Notably, not only did we observe higher basal expression of MAIT cell cytotoxicity markers in ALD than controls, but among ALD patients we also observed a small trend for stronger bacteria-induced MAIT cell cytotoxicity in those with increased plasma markers of bacterial translocation. Microarray dataset results are generally not limited to a single cell type only but extend to the investigated tissue/ organ as a whole. For this reason, we believe that further studies are required to clarify the migration patterns and expression of homing receptors on blood, gut and liver MAIT cells specifically, in the context of alcoholic liver disease.

We did not detect MAIT-specific TCR_V $\alpha 7.2 /$ TRAV1-2 transcripts in colon pinch biopsies of patients with ARC. The difficulty of obtaining both hepatic and intestinal tissue in patients with ALD, due to the issues of deranged coagulation and significant ascites that are found in many of these patients, precluded the in-depth analysis of intestinal MAIT cells, in particular in patients with SAH. We cannot exclude extrahepatic accumulation of MAIT cells in the mesenteric lymph nodes, where leaked bacterial products and bacteria-loaded mucosal antigen-presenting cells are likely to concentrate prior to systemic distribution. 35363940

MAIT cells from patients with ALD exhibited significantly compromised antibacterial cytokine and cytotoxic functions particularly with loss of IL-17, which plays an essential role in controlling bacterial dissemination and actively initiating the antibacterial response cascade ${ }^{50}$ Loss of this function could incapacitate localised and systemic host immunity to bacterial infection and is especially relevant to $E$. coli infection, which is one of the most common bacterial species found in cirrhotics. $^{23}$

Transcription factors including ROR $\gamma \mathrm{t}$, PLZF, Eomes and T-bet are strongly expressed in healthy MAIT cells ${ }^{51}$ and drive the acquisition of their innate-like/type-17 functional features, including mucosal and liver homing. ${ }^{52-55}$ The loss of antibacterial activity in our patients was found to be associated with loss of these transcription factors (ROR $\gamma t$ and PLZF). Analogous findings were reported in $\mathrm{HIV}^{51}$ and we hypothesise various mechanisms for this dysfunctional profile in ALD.

First, it is known that in vitro long-term bacterial stimulation can suppress MAIT cell expression of ROR $\gamma t$ and PLZF, ${ }^{51}$ suggesting that strong/chronic exposure to microbial antigens in pathological conditions in vivo could be responsible for this phenomenon, driving MAIT cells into 'poised/stunted' activation. This corresponds to our observations in ALD, which were always more pronounced in patients with more severe disease, 

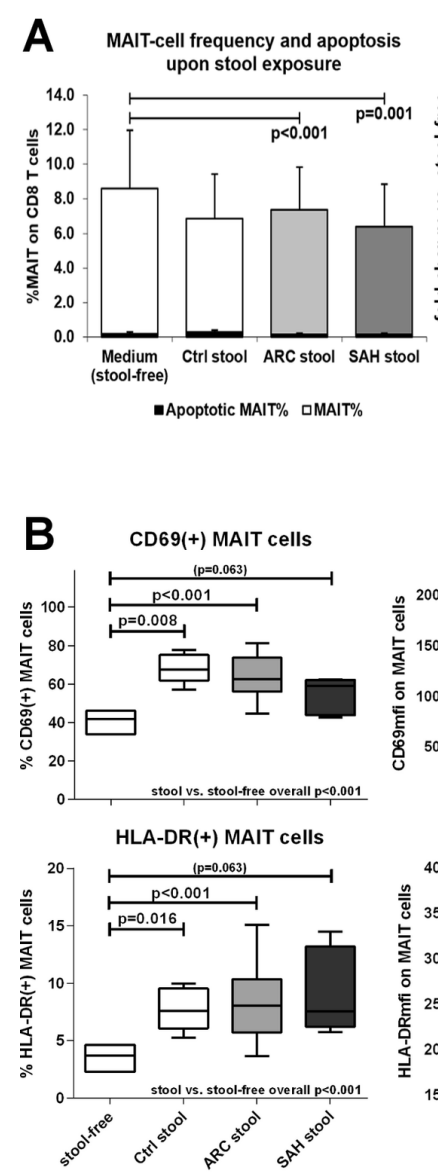
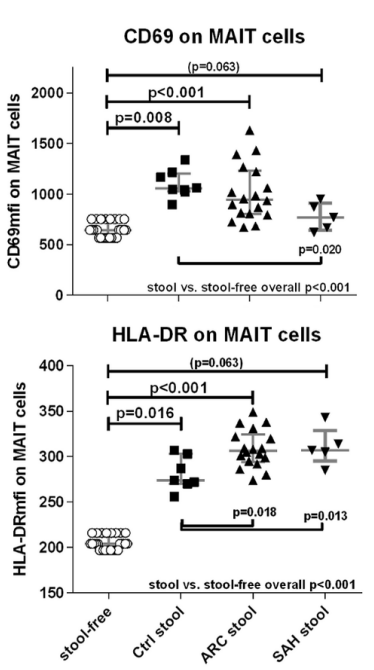
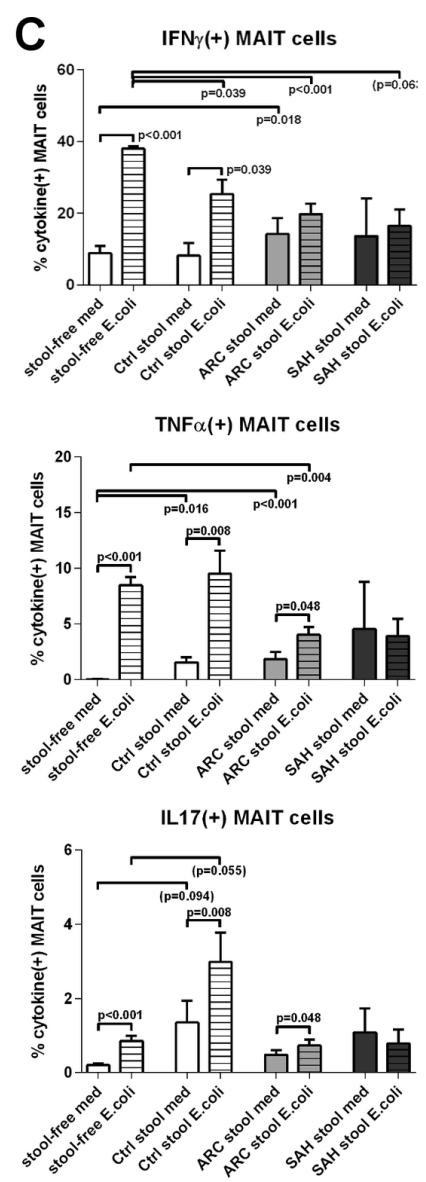
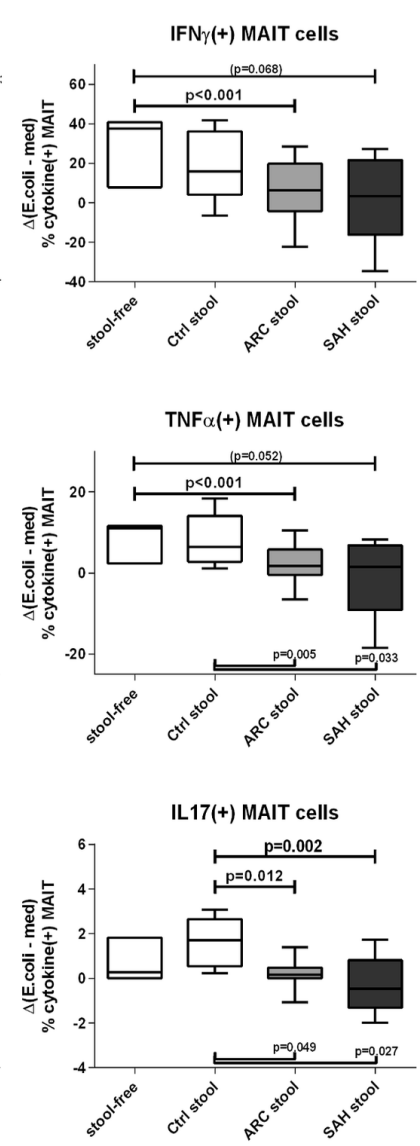
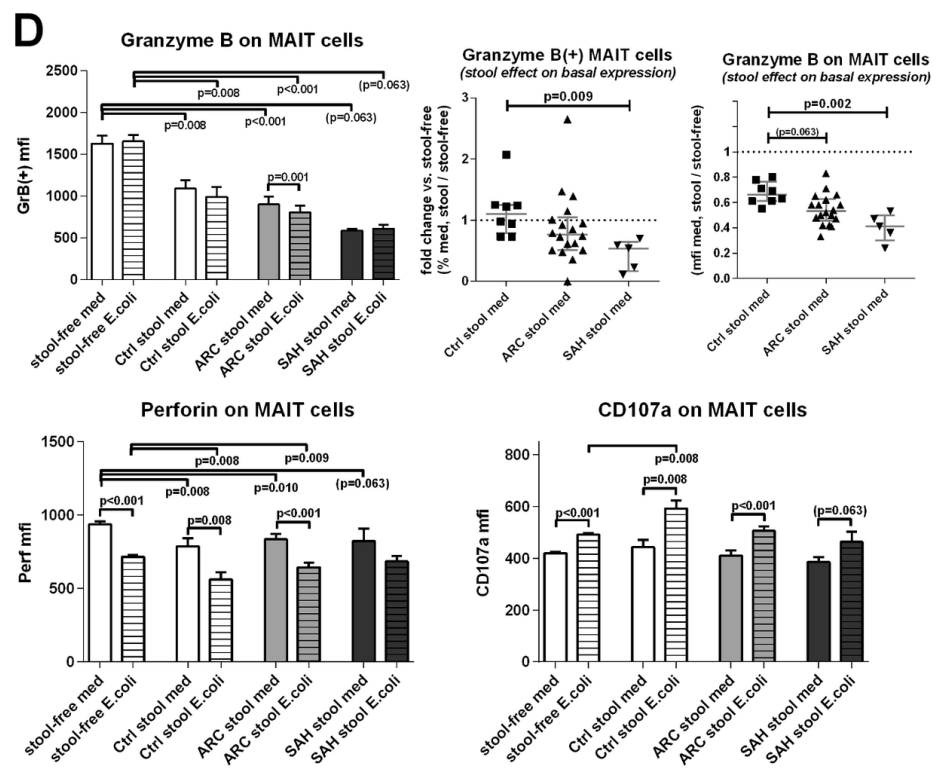

Figure 6 Contact between healthy PBMC and faecal extracts of bacterial antigens, toxins and metabolites (FEB) from ALD patients and controls causes quantitative and functional impairments of MAIT. (A) FEB-induced MAIT cell depletion. Apoptosis rates are not different between the groups, illustrating that FEB-induced MAIT cell depletion is apoptosis independent. Control stools: $n=12 ; A R C$ stools: $n=20$; SAH stools: $n=7$. The bar plot represents mean \pm SD. The black bars represent $\%$ of apoptotic VybrantFAM(+) MAIT cells; the white/shaded bars represent MAIT cell frequencies; both quantities are measured on the total CD8 T cell population, as described in 'Materials and Methods'. (B) FEB-induced hyperactivated state on MAIT, CD69/HLA-DR. (C) FEB-induced immunoinhibitory checkpoint upregulation, PD1/TIM3/LAG3. FEB-induced suppression of MAIT cell antibacterial cytokine (D) and cytotoxic (E) responses; zebra bars represent Escherichia coli-stimulated results. In panels $B-E$, control stools: $n=8$; $A R C$ stools: $n=18$; SAH stools: $n=5$. ALD, alcohol-related liver disease; ARC, alcohol-related cirrhosis; MAIT, mucosa-associated invariant T cells; PMBC, peripheral blood mononuclear cells; SAH, severe alcoholic hepatitis. 
where plasma concentrations of markers of bacterial translocation and dissemination were higher.

Second, blood MAIT cells in our patients with ALD displayed increased CD69 expression, in line with published literature. ${ }^{7-11}$ Raised CD69 expression on MAIT cells could be linked to encountering gut bacteria ${ }^{33}$ and is relevant for lymphocyte enterohepatic recirculation, ${ }^{8} 2333$ with likelier hyperexpression on MAIT cells from ALD than controls, as contact with gut bacteria may be more substantial in patients with increased gut leakiness. Furthermore, CD69 hyperexpression is known to render $\mathrm{T}$ cells more susceptible to CD69:Gal1-mediated suppression of innate-like/type-17 transcription factors and responses. ${ }^{32-34}$ Notably, in our analysis, Gal1 expression was significantly higher in SAH liver tissue than controls, and it has been shown that intrahepatic MAIT cells express increased levels of CD69 in a diverse range of liver diseases. ${ }^{8}$

Lastly, it is known that adult MAIT cells are partially unresponsive to TCR-dependent stimulation without secondary cytokine signals, thus undergoing 'poised' functional activation. ${ }^{56}$ One of the most potent MAIT-stimulating cytokines, IL-7, induced MAIT cell functional restoration in $\mathrm{HIV}^{51}$ reactivating MAIT cell proliferation and cytokine production, including IL-17. ${ }^{1451}$ IL-7 is relevant to liver disease, being one of the first and strongest cytokines produced by hepatocytes during liver inflammation. ${ }^{57}$ However, in our study, blood MAIT cells expressed IL-7R (CD127) regardless of disease status, suggesting IL-7 as potential novel immunotherapeutic target for MAIT cell functional rescue in ALD. IL-15 is also known to enhance MAIT cell activity, although less powerfully. ${ }^{565859}$ Whether this difference may be therapeutically advantageous requires further study, but reprogramming MAIT cells with IL-15 could boost their antibacterial activities while potentially avoiding maximal activation and overwhelming MAIT-mediated tissue damage.

In our study, patients with SAH had the strongest MAIT cell loss and dysfunction and a greater frequency of acquired bacterial infection compared with ARC. The susceptibility to bacterial infection in our patients was also potentially underestimated, as most $\mathrm{SAH}$ were treated prophylactically with antibiotics on hospital admission and recruitment. This association is also supported by studies from Grimaldi $\mathrm{et}_{\mathrm{al}}{ }^{7}$, who show that propensity to bacterial infections is inversely correlated to MAIT cell counts. We believe that MAIT cell defects may be important for the susceptibility to infection observed in ALD, responsible for increased morbidity and mortality in these patients. ${ }^{2}{ }^{3}$ Furthermore, we believe that MAIT cells provide an important link to the intestinal immunity, which has to date remained elusive in this disease.

The state of immunoparesis characteristic of patients with ALD, especially patients with SAH, may be intensified by the combination of quantitative MAIT cell depletion and functional defects associated with the residual MAIT cell population. Ex vivo functionally reprogrammed MAIT cells, ${ }^{1451}$ MAIT cells derived from induced pluripotent stem cells (iPSC-derived MAIT cells $)^{60}$ and strategies aimed at restoring the gut barrier may represent novel attractive immunotherapeutic avenues for cirrhosis and alcoholic liver disease.

\footnotetext{
Author affiliations

1 Institute of Hepatology London, Foundation for Liver Research, London, UK ${ }^{2}$ Division of Transplantation, Immunology and Mucosal Biology, Faculty of Life Sciences and Medicine, King's College London, London, UK

${ }^{3}$ Institute of Liver Studies, King's College London, London, UK

${ }^{4}$ Peter Medawar Building for Pathogen Research, University of Oxford, Oxford, UK ${ }^{5}$ Centre for Liver Research and NIHR BRU in Liver Disease, University of Birmingham, Birmingham, UK
}

${ }^{6}$ Department of Gastroenterology, Basildon University Hospital, Basildon, UK ${ }^{7}$ Department of Gastroenterology, Royal Berkshire Hospital, Reading, UK

${ }^{8}$ Department of Gastroenterology, Hepatology and Nutrition, Virginia Commonwealth University and McGuire VAMC, Richmond, Virginia, USA

${ }^{9}$ Department of Biomedical Sciences, University of Westminster, London, UK

${ }^{10}$ Department of Gastroenterology, Military Medical Academy, Sofia, Bulgaria

Contributors AR: study design, experimental work, data acquisition and analysis, manuscript preparation. SC: study design and supervision, manuscript preparation and final approval. Experimental collaborators: AK, HCJ, PK and YO. Provision of samples: VinP, SA, JAW, ST, GW, JMR, DS, AE, AF, JSB, KM, CL, VisP, TH, SP, MS and KK. Critical evaluation of the manuscript for important intellectual content and study funding: RW. All authors reviewed and approved the final version of the manuscript.

Funding This study was funded by the Foundation for Liver Research.

Competing interests None declared.

Patient consent Detail has been removed from this case description/these case descriptions to ensure anonymity. The editors and reviewers have seen the detailed information available and are satisfied that the information backs up the case the authors are making.

Ethics approval All local recruiting centres had individual ethical approval: the North East London Research Ethics Committee (08/H0702/52); the National Research Ethics Service (NRES) Committees South West (13/SW/0219), Portsmouth South Central (12/SC/0359), London Riverside (135979), London Westminster (12/L0/1417); the Local Research Ethics Committee (LREC) South Birmingham (98/CA5192 and 06/Q2708/11); the Steroids or Pentoxifylline for Alcoholic Hepatitis trial (STOPAH) (09/MRE09/59); and the Richmond VA Medical Center IRB (bajaj004).

Provenance and peer review Not commissioned; externally peer reviewed.

Open Access This is an Open Access article distributed in accordance with the Creative Commons Attribution Non Commercial (CC BY-NC 4.0) license, which permits others to distribute, remix, adapt, build upon this work non-commercially, and license their derivative works on different terms, provided the original work is properly cited and the use is non-commercial. See: http://creativecommons.org/ licenses/by-nc/4.0/

(C) Article author(s) (or their employer(s) unless otherwise stated in the text of the article) 2018. All rights reserved. No commercial use is permitted unless otherwise expressly granted.

\section{REFERENCES}

1 Albillos A, Lario M, Álvarez-Mon M. Cirrhosis-associated immune dysfunction: distinctive features and clinical relevance. J Hepatol 2014;61:1385-96.

2 Bonnel AR, Bunchorntavakul C, Reddy KR. Immune dysfunction and infections in patients with cirrhosis. Clin Gastroenterol Hepatol 2011;9:727-38.

3 Louvet A, Mathurin P. Alcoholic liver disease: mechanisms of injury and targeted treatment. Nat Rev Gastroenterol Hepatol 2015;12:231-42.

4 Louvet A, Wartel F, Castel H, et al. Infection in patients with severe alcoholic hepatitis treated with steroids: early response to therapy is the key factor. Gastroenterology 2009; 137:541-8

5 Markwick LJ, Riva A, Ryan JM, et al. Blockade of PD1 and TIM3 restores innate and adaptive immunity in patients with acute alcoholic hepatitis. Gastroenterology 2015;148:590-602

6 Fernández J, Bert F, Nicolas-Chanoine MH. The challenges of multi-drug-resistance in hepatology. J Hepatol 2016;65:1043-54.

7 Grimaldi D, Le Bourhis L, Sauneuf B, et al. Specific MAIT cell behaviour among innatelike T lymphocytes in critically ill patients with severe infections. Intensive Care Med 2014;40:192-201.

8 Jeffery HC, van Wilgenburg B, Kurioka A, et al. Biliary epithelium and liver B cells exposed to bacteria activate intrahepatic MAIT cells through MR1.J Hepatol 2016;64:1118-27.

9 Hinks TS. Mucosal-associated invariant T cells in autoimmunity, immune-mediated diseases and airways disease. Immunology 2016;148:1-12.

10 Reantragoon R, Boonpattanaporn N, Corbett AJ, et al. Mucosal-associated invariant T cells in clinical diseases. Asian Pac J Allergy Immunol 2016;34:3-10.

11 Kurioka A, Walker LJ, Klenerman P, et al. MAIT cells: new guardians of the liver. Clin Trans/ Immunology 2016;5:e98.

12 Lee OJ, Cho YN, Kee SJ, et al. Circulating mucosal-associated invariant T cell levels and their cytokine levels in healthy adults. Exp Gerontol 2014;49:47-54

13 Dusseaux M, Martin E, Serriari N, et al. Human MAIT cells are xenobiotic-resistant, tissue-targeted, CD161hi IL-17-secreting T cells. Blood 2011;117:1250-9.

14 Tang XZ, Jo J, Tan AT, et al. IL-7 licenses activation of human liver intrasinusoidal mucosal-associated invariant T cells. J Immunol 2013;190:3142-52.

15 Corbett AJ, Eckle SB, Birkinshaw RW, et al. T-cell activation by transitory neo-antigens derived from distinct microbial pathways. Nature 2014;509:361-5. 
16 McWilliam HE, Eckle SB, Theodossis A, et al. The intracellular pathway for the presentation of vitamin B-related antigens by the antigen-presenting molecule MR1. Nat Immunol 2016;17:531-7.

17 Ussher JE, van Wilgenburg B, Hannaway RF, et al. TLR signaling in human antigenpresenting cells regulates MR1-dependent activation of MAIT cells. Eur J Immunol 2016;46:1600-14.

18 Reantragoon R, Corbett AJ, Sakala IG, et al. Antigen-loaded MR1 tetramers define $T$ cell receptor heterogeneity in mucosal-associated invariant T cells. J Exp Med 2013;210:2305-20.

19 Jo J, Tan AT, Ussher JE, et al. Toll-like receptor 8 agonist and bacteria trigger potent activation of innate immune cells in human liver. PLoS Pathog 2014;10:e1004210.

20 Le Bourhis L, Dusseaux M, Bohineust A, et al. MAIT cells detect and efficiently lyse bacterially-infected epithelial cells. PLoS Pathog 2013;9:e1003681.

21 Young MH, U'Ren L, Huang S, et al. MAIT cell recognition of MR1 on bacterially infected and uninfected cells. PLoS One 2013;8:e53789.

22 Salerno-Goncalves R, Rezwan T, Sztein MB. B cells modulate mucosal associated invariant T cell immune responses. Front Immunol 2014;4:511.

23 Booth JS, Salerno-Goncalves R, Blanchard TG, et al. Mucosal-Associated Invariant T Cells in the Human Gastric Mucosa and Blood: Role in Helicobacter pylori Infection. Front Immunol 2015;6:466

24 Kurioka A, Ussher JE, Cosgrove C, et al. MAIT cells are licensed through granzyme exchange to kill bacterially sensitized targets. Mucosal Immunol 2015;8:429-40.

25 Maddrey WC, Boitnott JK, Bedine MS, et al. Corticosteroid therapy of alcoholic hepatitis. Gastroenterology 1978;75:193-9.

26 Riva A, Laird M, Casrouge A, et al. Truncated CXCL10 is associated with failure to achieve spontaneous clearance of acute hepatitis C infection. Hepatology 2014;60:487-96.

27 Grootjans J, Thuijls G, Verdam F, et al. Non-invasive assessment of barrier integrity and function of the human gut. World J Gastrointest Surg 2010;2:61-9.

28 Van Rhijn I, Kasmar A, de Jong A, et al. A conserved human T cell population targets mycobacterial antigens presented by CD1b. Nat Immunol 2013;14:706-13.

29 Tilloy F, Treiner E, Park SH, et al. An invariant T cell receptor alpha chain defines a novel TAP-independent major histocompatibility complex class lb-restricted alpha/ beta T cell subpopulation in mammals. J Exp Med 1999;189:1907-21.

30 Affò S, Dominguez M, Lozano JJ, et al. Transcriptome analysis identifies TNF superfamily receptors as potential therapeutic targets in alcoholic hepatitis. Gut 2013:62:452-60.

31 Sharma PK, Wong EB, Napier RJ, et al. High expression of CD26 accurately identifies human bacteria-reactive MR1-restricted MAIT cells. Immunology 2015;145:443-53.

32 de la Fuente H, Cruz-Adalia A, Martinez Del Hoyo G, et al. The leukocyte activation receptor CD69 controls T cell differentiation through its interaction with galectin-1. Mol Cell Biol 2014;34:2479-87.

33 Radulovic K, Niess JH, Radulovic K, et al. CD69 Is the Crucial Regulator of Intestinal Inflammation: A New Target Molecule for IBD Treatment?, CD69 Is the Crucial Regulator of Intestinal Inflammation: a new target molecule for IBD Treatment? J Immunol Res 2015:e497056.

34 Yakushina VD, Vasil'eva OA, Ryazantseva NV et al. The effects of galectin-1 on the gene expression of the transcription factors TBX21, GATA-3, FOXP3 and RORC. Mol Cell Biochem 2015;398:245-9.

35 Rao R. Endotoxemia and gut barrier dysfunction in alcoholic liver disease. Hepatology 2009;50:638-44.

36 Patel S, Behara R, Swanson GR, et al. Alcohol and the Intestine. Biomolecules 2015;5:2573-88.

37 Canesso MCC, Lacerda NL, Ferreira CM, et al. Comparing the effects of acute alcohol consumption in germ-free and conventional mice: the role of the gut microbiota. BMC Microbiol 2014;14:240
38 Leclercq S, Matamoros S, Cani PD, et al. Intestinal permeability, gut-bacterial dysbiosis, and behavioral markers of alcohol-dependence severity. Proc Natl Acad Sci USA 2014;111:E4485-93.

39 Balmer ML, Slack E, de Gottardi A, et al. The liver may act as a firewall mediating mutualism between the host and its gut commensal microbiota. Sci Trans/ Med 2014;6:237ra66.

40 Tuomisto S, Pessi T, Collin P, et al. Changes in gut bacterial populations and their translocation into liver and ascites in alcoholic liver cirrhotics. BMC Gastroenterol 2014;14:40.

41 Dias J, Sobkowiak MJ, Sandberg JK, et al. Human MAIT-cell responses to escherichia coli: activation, cytokine production, proliferation, and cytotoxicity. J Leukoc Biol 2016;100:233-40.

42 Cosgrove C, Ussher JE, Rauch A, et al. Early and nonreversible decrease of CD161++ I MAIT cells in HIV infection. Blood 2013;121:951-61.

43 Inoue $\mathrm{H}$, Tani K. Multimodal immunogenic cancer cell death as a consequence of anticancer cytotoxic treatments. Cell Death Differ 2014;21:39-49.

44 Li M, He Y, Zhou Z, et al. MicroRNA-223 ameliorates alcoholic liver injury by inhibiting the IL-6-p47(phox)-oxidative stress pathway in neutrophils. Gut 2017;66:705-15.

45 Eggers $\mathrm{V}$, Pascher $\mathrm{A}$, Althoff $\mathrm{H}$, et al. Immune reactivity is more suppressed in patients with alcoholic liver disease than in patients with virus-induced cirrhosis after $\mathrm{CRH}$ stimulation. Alcohol Clin Exp Res 2006;30:140-9.

46 Leeansyah E, Ganesh A, Quigley MF, et al. Activation, exhaustion, and persistent decline of the antimicrobial MR1-restricted MAIT-cell population in chronic HIV-1 infection. Blood 2013:121:1124-35.

47 Eksteen B, Miles AE, Grant AJ, et al. Lymphocyte homing in the pathogenesis of extraintestinal manifestations of inflammatory bowel disease. Clin Med 2004;4:173-80.

48 Tacke F, Zimmermann HW, Berres ML, et al. Serum chemokine receptor CXCR3 ligands are associated with progression, organ dysfunction and complications of chronic liver diseases. Liver Int 2011;31:840-9.

49 Dominguez M, Miquel R, Colmenero J, et al. Hepatic expression of CXC chemokines predicts portal hypertension and survival in patients with alcoholic hepatitis. Gastroenterology 2009;136:1639-50.

50 Curtis MM, Way SS. Interleukin-17 in host defence against bacterial, mycobacterial and fungal pathogens. Immunology 2009;126:177-85.

51 Leeansyah E, Svärd J, Dias J, et al. Arming of MAIT cell cytolytic antimicrobial activity Is induced by IL-7 and defective in HIV-1 infection. PLOS Pathog 2015;11:e1005072.

52 Maggi L, Santarlasci V, Capone M, et al. CD161 is a marker of all human IL-17producing T-cell subsets and is induced by RORC. Eur J Immunol 2010;40:2174-81.

53 Kovalovsky D, Uche OU, Eladad S, et al. The BTB-zinc finger transcriptional regulator PLZF controls the development of invariant natural killer T cell effector functions. Nat Immunol 2008;9:1055-64.

54 Intlekofer AM, Takemoto N, Wherry EJ, et al. Effector and memory CD8+ T cell fate coupled by T-bet and eomesodermin. Nat Immunol 2005;6:1236-44.

55 Yang Y, Xu J, Niu Y, et al. T-bet and eomesodermin play critical roles in directing T cell differentiation to Th1 versus Th17. J Immunol 2008;181:8700-10.

56 Slichter CK, McDavid A, Miller HW, et al. Distinct activation thresholds of human conventional and innate-like memory T cells. JCI Insight 2016;1:e86292.

57 Sawa Y, Arima Y, Ogura H, et al. Hepatic interleukin-7 expression regulates T cell responses. Immunity 2009;30:447-57.

58 Jiang J, Yang B, An H, et al. Mucosal-associated invariant T cells from patients with tuberculosis exhibit impaired immune response. J Infect 2016;72:338-52.

59 Sattler A, Dang-Heine C, Reinke P, et al. IL-15 dependent induction of IL-18 secretion as a feedback mechanism controlling human MAIT-cell effector functions. Eur J Immunol 2015;45:2286-98.

60 Sugimoto C, Fujita H, Wakao H. Mucosal-associated invariant T cells from induced pluripotent stem cells: A novel approach for modeling human diseases. World J Stem Cells 2016:8:158-69. 